\title{
Überlasteter Richter wegen Strafvereitelung angeklagt
}

\section{Einleitung:}

Das höchst rechtsstaatliche und ausdifferenzierte deutsche Strafrechtssystem genießt weltweit einen äußerst guten Ruf. Viele Länder in der Welt haben es kopiert und beziehen sich fortlaufend auf die Entwicklung der StGB- und StPO-Dogmatik. Es gibt aber viele Anlässe, alarmiert zu fragen, ob die Substanz dieses guten Rufes nicht seit längerem einer Erosion unterliegt, die es peinlich erscheinen lässt, sich diesen weiterhin in der internationalen Selbstdarstellung zu Gute zu halten. Einen solchen Anlass mit tieferer Bedeutung sehe ich in dem seit $200 \mathrm{I}$ eskalierten Konflikt zwischen der Staatsanwaltschaft und Richter S. in Heidelberg.

\section{Was ist passiert?}

Über den Konflikt ist mittlerweile in Fachpresse und anderen Medien verschiedentlich berichtet worden. Zur Erinnerung: Mit Anklageschrift vom I7. I 2. 200 I erhob die Staatsanwaltschaft Heidelberg gegen den damals 63 -jährigen Richter S. wegen Strafvereitelung im Amt Anklage gem. $\int \mathbb{S} 258,258 \mathrm{a}$ StGB. Der Vorwurf lautete, er habe in 2 I Fällen als Jugendrichter »ohne Anzeige einer vermeintlichen Überlastung bewusst ... Verfahren mit dem Willen, diese für eine längere Zeit nicht zu bearbeiten, nicht ordnungsgemäß gefördert ... und dabei als sichere Folge seines Untätigbleibens vorausgesehen, dass dadurch der staatliche Strafanspruch auf geraume Zeit nicht durchgesetzt werden kann."Mit Beschluss vom 17.04.2003 lehnte das LG die Eröffnung des Hauptverfahrens aus tatsächlichen und rechtlichen Gründen ab (Deutsche Richterzeitung Sept. 2004, 26I und Betrifft Justiz Sept. 2004, 340). Auf die Sofortige Beschwerde der Staatsanwaltschaft vom 02.05. 2003 hin bestätigte das OLG Karlsruhe mit Beschluss vom 09. I 2. 2003 in vollem Umfang den Beschluss des LG. Mittlerweile hatte sich der Richter S. aufgrund der persönlichen Belastung vorzeitig pensionieren lassen. Von diesem Verlauf sich betroffen fühlende Richterkolleginnen und -kollegen brachten die Sache ans Licht von Öffentlichkeit und Politik, von Fachverbänden und Fachpresse. Am 3 I. 8. 2004 stellte ein Richterkollege gegen den die Anklage verantwortenden Staatsanwalt Strafanzeige wegen Verdachts der Verfolgung Unschuldiger $(\mathbb{S} 344$ StGB). Die entsprechende Ablehnung einer Aufnahme der Ermittlungen durch die Staatsanwaltschaft Heidelberg vom 16.09. 2004 wurde auf die Gegenvorstellung des anzeigenden Richters hin durch die Generalstaatsanwaltschaft am 22.9.2004 bestätigt. Die Partei der GRÜNEN stellte am 07. I0. 2004 eine ausführliche parlamentarische Anfrage, in der auch Auskünfte betreffend andere problematische, augenscheinlich die richterliche Unabhängigkeit beeinträchtigende Vorgänge verlangt wurden. Diese wurde durch Stellungnahme des Justizministers Goll - ausgerechnet ein Politiker der ehemaligen »Rechtsstaatspartei« FDP - von Baden-Württemberg vom 26. I0. 2004 dahingehend beantwortet, dass es sich um ein ganz normales Strafverfahren gehandelt habe, bei dem aufgrund »unklarer Rechtslage « eine Anklage »rechtlich vertretbar« gewesen sei (Drucksache I 3/3629). Auf die anderen Aspekte wurde darin nicht eingegangen. Auch die Generalstaatsanwaltschaft stellte sich vor den Ankläger. Fachverbände wandten 
sich entschieden gegen das, was als Beeinträchtigung der richterlichen Unabhängigkeit gewertet wurde (Amtsrichterverband am i6. I I., Neue Richter-Vereinigung am 22. I I. 2004).

Zu den strafrechtsdogmatischen, justiz- und kriminalpolitischen Aspekten hat Kollege Peter Alexis Albrecht in seinem in der ZRP (H. 8/2004, 259) veröffentlichten Essay umfassend Stellung genommen. Dem kann ich mich vorbehaltlos anschließen. Strafrechtsdogmatisch ist hier nicht weiter zu debattieren, so hatten zuvor schon LG und OLG klargestellt. Ich möchte deshalb hier einen Versuch anbieten, diesen Fall einer Eskalation auf der Ebene des von der Rechtssoziologie so genannten »second code « zu verstehen, es als » hidden curriculum « oder »informelles Programm« zu interpretieren: Welche » wirklichen Gründe «, welche unterschwelligen, wahrscheinlich unbewussten psychischen und psychosozialen Motive und Gesetzmäßigkeiten haben hier im Gewande einer strafrechtlichen Argumentation gewirkt?

\section{Sachfremde Affekte in der Anklageschrift}

Schon die Länge der 3 I-seitigen Anklageschrift mit dem Tatvorwurf der »versuchten und vollendeten Strafvereitelung im Amt « in 2 I detailliert dargestellten »Fällen « ist interpretationswürdig. Die Normalität der Struktur einer Anklageschrift und die routinemäßigen Formulierungen - es könnten teilweise die häufig benutzten Textbausteine aus dem PC sein - bewirken in der Wahrnehmung nicht nur Befremden, sondern auch die Assoziation mit gefährlichen Serientätern. Auch der vorwurfsvoll klingende Hinweis, der Angeschuldigte habe zu seinen persönlichen Verhältnissen keine ausführlichen Angaben gemacht, belegt eine Technik, die Dinge aus dem sozialen Kontext zu reißen und mit Bösem zu assoziieren. Dasselbe ergibt sich aus der entwertenden Zuschreibung, die vom »Angeschuldigten « geltend gemachte Überlastung sei nur eine »vermeintliche« gewesen. Sachfremd vorgeworfen wird gleich am Anfang der Anklageschrift, der Beschuldigte habe Abteilungsleiter der Staatsanwaltschaft in diesem Schriftsatz persönlich angegriffen. Ein Affekt klingt auch in der Formulierung an, der »Angeschuldigte « habe der Vorladung zur Vernehmung keine Folge geleistet und sich erst »nach erfolgter Akteneinsicht « zur Sache eingelassen, und zwar »ähnlich zögerlich, wie er die aufgeführten Verfahren bearbeitet hat « (S. I 8 der Anklageschrift). Sicherlich verbergen sich Affekte in vielfältiger Weise in jeglicher Berufsausübung. Hier jedoch haben sie eine besondere Bedeutung und bedürfen der Interpretation.

Dasselbe gilt für die Unverblümtheit der strafrechtlichen Fehlwertung, der schlichtweg unvertretbaren Auslegung und Subsumtion (so auch Albrecht ZRP 2004, 259). Schon einem Studierenden im 3. Semester würde in der Klausur angekreidet, was der Ankläger auf S. 22 zum Verhältnis von Tun und Unterlassen meint. Mit perseveranter Ignoranz wird die - auch vom LG und OLG in den Nichteröffnungsbeschlüssen offenbar nur mit Kopfschütteln entgegen gehaltene - Sperrwirkung des Rechtsbeugungstatbestandes $(\$ 339$ StGB) gegenüber der Strafvereitelung im Amt $(\mathbb{2} 258 \mathrm{a}$ StGB) auch noch in der Sofortigen Beschwerde aufrecht erhalten. Angesichts der ansonsten äußerst ausführlichen und routinierten Anklageschrift ist dies kaum mit verzeihlicher Flüchtigkeit zu erklären. Man kann hier nicht mehr einfach von einem »Vergaloppieren « eines vereinzelten und vielleicht frustrierten Staatsanwalts sprechen, der sich in die Eskalation hat treiben lassen. 
Es klingt nicht so, als ob die Anklage wirklich ernst gemeint war. Die Staatsanwaltschaft muss schon allein deshalb mit Ablehnung der Eröffnung gerechnet haben, weil nach dem »Krähenprinzip« ein Richterkollege sicherlich nicht ohne weiteres desavouiert wird. Das ganze sieht nach einer gezielten Provokation aus, nach mehr als nur einem Nadelstich. Er erscheint als bewusste Inszenierung, als Muskel-Zeigen in einem Machtkampf.

Angenommen werden muss, dass hier ein verselbständigtes Zweckdenken am Werke war, welches sich deshalb so »nass-forsch« Bahn brechen konnte, weil ein Gefühl der Sicherheit und Unanfechtbarkeit dahinter stand, möglicherweise das affektiv unterfütterte satte Bewusstsein: »Die >großen Brüder General und Justizminister stehen hinter mir! « Dieses Gefühl, eine möglicherweise nicht einmal bewusste Kollusion zwischen Minister und Staatsanwaltschaft, kann wiederum auf einem abstrakten und allgemeinen Zeitgeist neo-feudaler, exekutivischer Selbstherrlichkeit ebenso beruhen wie auf einer sehr konkreten und spezifischen Disziplinierungstendenz der Generalstaatsanwaltschaft und des Justizministeriums gegenüber der Dritten Gewalt im Lande. Im Ergebnis jedenfalls ist es als Versuch zu verstehen, die Balance zwischen den drei Gewalten performativ zu verändern. Sowohl der ausführende Staatsanwalt als auch die ihm den Rücken stärkenden Kräfte mögen unbewusst dabei von einer gewissen narzisstischen Macht-Lust getragen gewesen sein, wie sie durch Erhabenheit über inhaltliche Kritik und faktische Kontrolle ermöglicht wird. Der narzisstische Gewinn ermöglicht das Überspielen von Schamgefühlen wegen der exponierten Unkenntnis oder Missachtung des Rechts ebenso wie er Einfühlung und Schuldgefühle gegenüber dem Richterkollegen, der ja sozusagen unter demselben Dach haust, blockiert. Zum Ausdruck kommen darin möglicherweise im Sinne einer immer latenten institutionellen Rivalität auch langfristig gehegte Affekte des Grolls und des Neides gegenüber den unabhängigeren Richterkollegen. Bezeichnend erscheint auch, dass die einzigen höchstrichterlichen Entscheidungen betreffend Strafvereitelung im Amt, auf die sich die Anklage zur Untermauerung der Subsumtion des Vorsatzes bezieht, Staatsanwälte betrafen und den Vorsatz gerade verneinten. Auch in der ganz konträren Auslegung dieser Urteile kann man ein Kompensations- oder gar Rachebedürfnis erkennen. Auch eine Projektion der eigenen Gefühle von Schwäche und Ärger angesichts strukturell gewachsener Überlastung, eine Verschiebung weg von den eigentlichen Urhebern derselben sowie entsprechende Verleugnungsmechanismen mögen dazu beigetragen haben.

Solche universellen Projektions- und Übertragungsmechanismen, die sich in nahezu jeder Institution und jedem Arbeitszusammenhang abspielen, die im alltäglichen »Mobbing « zum Ausdruck kommen, sie erklären meines Erachtens diesen Vorgang noch nicht ausreichend. Es sind wahrscheinlich höchst reale sozio-ökonomische Interessen, welche sich so sozialpsychologische Mechanismen zu Nutze machen und den Weg bahnen. Man kann es so sehen: Die Achse Staatsanwalt-Generalstaatsanwalt-Justizminister repräsentiert eine gesellschaftliche Modernisierungstendenz, den Triumph der Exekutive und der Bürokratie. Der maßgebliche Hebel ist die Ökonomisierung, der Sparzwang als scheinbarer Sachzwang. Ebenso wie Verbrechensfurcht weitgehend ein die Realität nicht erfassendes Phänomen medialer und politischer Manipulation ist, so sind auch die Sachzwänge vielfach nur scheinbare, nämlich gemäß politischer Opportunität geschaffene Konstruktionen, Legitimation für machtstrukturell bedingte Ressourcenverteilung.

Dieser »Fall« erscheint in mehrfacher Hinsicht symptomatisch, und zwar über das hinaus, was schon von Albrecht (ZRP 2004, 259) als rechtsstaatlich prekär heraus- 
gestellt worden ist: die Suggestion, man könne durch eine sich immer weiter ausweitende strafrechtliche Kontrolle soziale Probleme und Risiken bewältigen. Die transparente, rechtsstaatliche und für die Demokratie essentielle Struktur der Gewaltenteilung hat auch eine wichtige sozial- und individual-psychologische Funktion: Sie trägt dazu bei, Vertrauen zu entwickeln und Ängste vor Bemächtigung zu verarbeiten. Durch Relativierung der Gewaltenteilung wird dieses Vertrauen ebenso untergraben wie durch die multiplen Manipulationstechniken heutiger Herrschaftsstrukturen. Gefördert werden dadurch gesellschaftliche und individuelle Regressionsprozesse der Polarisierung und Spaltung, der Ausgrenzung und Projektion des »Bösen «, des Bemühens um inhaltsleere Kontrolle und Kontingentierung des Bedrohlichen statt substantieller Anknüpfung an den empirischen Bedingungen sozialer Probleme.

Die »neue Unverschämtheit « einer Anklageerhebung in vollem Bewusstsein, dass sie symbolisch gemeint ist und andere Zwecke transportiert, trägt zu einer Verwahrlosung den Rechtsstaat sichernder Dogmatik bei. Dass dies von zentralen Staatsautoritäten quasi in Delegation mit getragen wird, bewirkt vielleicht einerseits den Jubel bestimmter Wählerschichten, untergräbt aber andererseits das Vertrauen und die Strukturen des diskursiv konzipierten Rechtsstaats. Es geht letztlich um die Durchbrechung der Gewaltenteilung, um Machtusurpation durch die Exekutive, um die destruktive Dynamik der Macht.

Damit soll nicht suggeriert werden, die Justiz sei ein ganz intakter Bereich gesellschaftlicher Machtverteilung: Besagte Sachzwänge und Ressourcenknappheiten, verbrämt als »Modernisierung «, »Qualitätssicherung « und »Effizienzsteigerung «, haben höchstwahrscheinlich faktisch längst zu oberflächlicher, weitgehend beziehungsloser Massenabfertigung, zu erheblichen Einbußen an rechtsstaatlicher Qualität geführt. Damit wird das Strafverfahren endgültig dysfunktional, selbst-destruktiv: Gerade die »materielle Wahrheit « (\244 II StPO), das Verstehen des in der Straftat zum Ausdruck kommenden sozio-ökonomischen und psycho-sozialen Konflikts in seiner Konkretheit, stellt eine wichtige Facette im Prozess gesellschaftlicher Konfliktverarbeitung und -bewältigung dar. Darin liegt eine Re-Inszenierung derjenigen psycho-sozialen Situation, welche in zunehmend vielen Fällen den Anfang und Verlauf von Fehl-Sozialisation, Kriminalität und Kriminalisierung kennzeichnet: Vernachlässigung, Nicht-Achtung, Beziehungslosigkeit, Entwertung, Ausgrenzung. Darin liegt auch ein Beitrag zur gesellschaftlichen Entfremdung. Die Maßgabe, diesen größeren sozialen und psycho-sozialen Kontext nicht zu reflektieren, spiegelt sich nicht zuletzt in der Engstirnigkeit der 1982 restaurierten Juristenausbildung, welche erst kürzlich wieder ein wenig in Richtung Interdisziplinarität und sozialer Realität geöffnet wurde.

Dass ein sich betroffen fühlender Richterkollege den Ankläger nun seinerseits wegen Verfolgung Unschuldiger $(\mathbb{S} 344 \mathrm{StGB})$ angezeigt hat, kann man das als Abstieg auf das Niveau der Staatsanwaltschaft und als erkennbar aussichtslosen Akt verzweifelter »beruflicher Notwehr« (so Albrecht ZRP 2004, 259) verstehen, weil die Staatsanwaltschaft mutmaßlich nicht anklagen wird. Gleichwohl sollte man sich vergegenwärtigen, dass die Anzeige eines Verbrechens grundsätzlich sorgfältige Ermittlungen gebietet. In der 3 I-seitigen Anklageschrift wird mangelnder Arbeitseinsatz vorgeworfen, ohne dass irgendwelche Feststellungen zur Arbeitsbelastung getroffen wurden. Diese Unterlassung allein würde normalerweise für den hinreichenden Tatverdacht genügen. Aber wenigstens wurde damit ein Signal gesetzt, das angesichts zunehmenden gegenseitigen Mobbings in Institutionen und Sozialbeziehungen in einer offenbar zunehmend entfremdeten und Rechtsstaatlichkeit verachtenden Gesellschaft aufrüttelt. 